\title{
EDITORIAL
}

\section{ENFERMERÍA DE CUIDADOS CULTURALES}

\section{Rachel Spector}

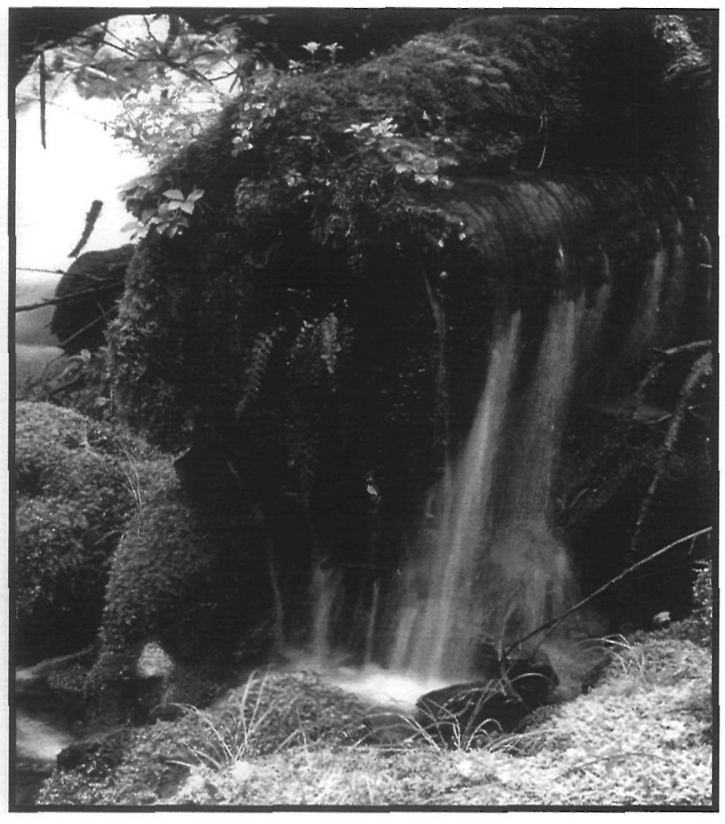

\section{INTRODUCCIÓN}

Hace poco vine a España de vacaciones prejubilación con mi marido. La estancia se convirtió en una increíble aventura de investigación y aprendizaje sobre las tradiciones respecto a la SALUD en este país. Visitamos varias ciudades, y llevé a mi marido a sitios en los que no hubiéramos estado de no ser por mi búsqueda de respuestas a las cuestiones que sobre la SALUD - fisica, mental y espiritual- se me plantean. Cuando hablo de SALUD (con mayúsculas) hago referencia a "el equilibrio de la persona (fisico, mental y espiritual) en sí, y con el mundo exterior (naturaleza, familia, comunidad y metafisica)".

Dicha visita me permitió explorar diversos lugares -hospitales, clínicas, farmacias, mercados, iglesias, museos... - desde el punto de vista de la SALUD en cada contexto. Indagué sobré el significado de este dinámico concepto. Los frutos de esta investigación aún he de "digerirlos" pero puedo afirmar que lo que he aprendido constituye sólo parte del enorme fenómeno de la enfermeria de cuidados culturales en España.

Como enfermeras, proporcionamos cuidados a personas de los más diversos ámbitos y antecedentes culturales. El cambio demográfico que se está produciendo en España con la llegada masiva de inmigrantes, traerá consigo sin duda el consiguiente cambio en las necesidades de SALUD de la población. Las enfermeras deberían ser el vinculo que traslade las creencias y prácticas del paciente al sistema de salud, y viceversa. Es esencial la comprensión del significado que el término SALUD posee para todas estas culturas. Y el momento para contribuir a esa comprensión ha llegado. Sin embargo, este conocimiento no se adquiere de la noche a la mañana. Existen multitud de factores interrelacionados que deben analizarse, debatirse, y tratarse para que nuestros conocimientos, actitudes y habilidades comiencen a cambiar. En nuestro recorrido hacia el entendimiento cultural, existen determinados conceptos que aprender y actividades que llevar a cabo.

En 1974, creé un curso de pre-grado, "La Cultura y los Cuidados de la Salud", que surgió a raíz de mi "ignorancia" sobre todos aquellos aspectos culturales de la salud y la enfermedad que afectan al proceso de socialización en el sistema de salud actual. El curso partió de una promesa que hice a un grupo de estudiantes en una clase en la que tomé conciencia de las numerosas diferencias culturales en las creencias y prácticas referentes a la salud y a la enfermedad. Me vi a mi misma, "la enfermera" a través de los ojos del paciente, la antítesis de la imagen que tenía hasta entonces.

En 1977 me animaron a escribir la primera edición de mi libro Cultural Diversity in Health and Illness (Diversidad Cultural en la Salud y la 
Enfermedad). El objetivo de este texto, que hoy día va por la $5^{\text {a }}$ edición, era concienciar al lector sobre las dimensiones y complejidades que implica la dispensación de cuidados de salud a personas de diversas procedencias culturales. El libro se basa en tres conceptos entrelazados:

- La consistencia hereditaria

- Las tradiciones de salud

- Los fenómenos culturales que afectan a la salud

\section{CONSISTENCIA HEREDITARIA}

Estés y Zitzow desarrollaron esta teoría en 1980 con objeto de describir "hasta qué punto el estilo de vida de una persona refleja su cultura tradicional". La teoría se ha ampliado con la intención de abarcar las diferentes culturas, ya sean europeas, asiáticas, africanas o hispanas (Spector, 2000). Los valores que indican consistencia hereditaria existen en un continuum, de manera que una misma persona puede poseer características tanto de herencia consistente (tradicional) como inconsistente (moderna o culturizada). El concepto de consistencia hereditaria implica la determinación de los vínculos de una persona con sus antecedentes étnicos, culturales y religiosos.

\section{MODELO DE TRADICIONES DE SALUD}

Este modelo emplea el concepto de SALUD holistica (que incluye el cuerpo, la mente y el espíritu) y analiza todo aquello que las personas hacen, desde una perspectiva tradicional, para prevenir la enfermedad, y restaurar, mantener y proteger la salud. El modelo, que comenzó en 1994 con la exposición sobre Tradiciones de salud de los inmigrantes en el museo Ellis Island de Nueva York, se ha ampliado con el tiempo.

Imaginemos la SALUD como un fenómeno complejo de varias facetas interrelacionadas, el equilibrio entre todas las de la persona. El cuerpo incluiría todos los aspectos físicos: herencia genética, edad, nutrición, condición física. La mente implicaría los procesos cognitivos o emocionales, tales como pensamientos, recuerdos, sentimientos, autodefensa y autoestima. La faceta espiritual abarcaría las prácticas espirituales aprendidas tanto positivas como negativas, los sueños, los símbolos, la intuición, la gracia y las fuerzas protectoras, y las fuerzas innatas o metafísicas positivas o negativas. Estas facetas se hallan en constante flujo y cambian continuamente, aunque cada una esté estrechamente relacionada con las demás y todas a su vez con el contexto de la persona, que incluye su familia, cultura, trabajo, comunidad, historia y ambiente.

\section{FENÓMENOS CULTURALES QUE AFECTAN A LA SALUD}

Giger y Davidhizar (1995) han identificado seis fenómenos culturales que varían según grupos culturales e influyen en la salud: control ambiental, variaciones biológicas, organización social, comunicación, espacio y orientación temporal. Estos seis fenómenos sirven para ilustrar no sólo la diversidad sino también la similitud que existe entre grupos culturales diferentes.

Estos conceptos están íntimamente relacionados. El dicho de "todo está relacionado con todo" subyace en la conexión de dichas facetas. Los aspectos relacionados con una dispensación de cuidados segura y eficaz representan el intento de ayudar a los profesionales a comprender lo que los inmigrantes o personas tradicionales experimentan en su interacción con ellos.

El concepto de Cuidados Culturales hace referencia a los cuidados de salud "culturalmente apropiados", "culturalmente sensibles" y "culturalmente competentes". ${ }^{1}$ (Spector, 2000, p.284). Los Cuidados Culturales se hacen necesarios a la hora de satisfacer las necesidades de un paciente, la familia y la comunidad. Se trata de "la provisión de cuidados de salud que supera las barreras culturales y sociales y tiene en consideración el contexto en el que el paciente vive al tiempo que la situación

\footnotetext{
${ }^{1}$ Culturalmente apropiado: el cuidador aplica los conocimientos que debe poseer para proporcionar al paciente el mejor cuidado posible. Culturalmente competente: en el contexto de los cuidados que proporciona, el cuidador comprende y tiene en cuanta el contexto global de la situación del paciente incluyendo factores como la inmigración, el estrés, la pobreza, y las diferencias culturales.

Culturalmente sensible: el cuidador posee conocimientos y actitudes constructivas hacia los diversos grupos culturales con los que trata.
} 
en la que le surgen sus problemas de salud". Abarcan tanto el contenido como el proceso. Los Cuidados Culturales analizan los aspectos relacionados con el paciente desde los dos lados del espejo: la cultura de salud dominante y la del paciente, su familia y la comunidad.

El contenido se encuentra tanto en la bibliografía de las ciencias de la salud como en la de otras disciplinas (sociología, antropología, folklore). El proceso es más ambiguo, puesto que implica la noción de que uno desarrolla la capacidad de "ver, escuchar y sentir" las profundamente arraigadas necesidades culturales de un paciente. Esto puede llegar a ser extremadamente complicado cuando las percepciones y opiniones del cuidador y el paciente difieren. Si leemos The spirit catches you and you fall down (El espíritu te atrapa y te caes)2, de Ann Fadiman (1977), comprendemos el sobrecogedor alcance de este dilema.

La tabla 1 expone la serie de pasos conceptuales a seguir, los contenidos específicos a aprender, las habilidades clave en relación a la competencia cultural, y las estrategias pedagógicas sugeridas. La progresión a seguir por el estudiante consistiría en:

1. Desarrollar sus conocimientos sobre la herencia y el papel de la cultura, la etnicidad, la religión, etc. en la interrelación entre personas de ideas tradicionales y modernas. Este paso lleva implícito que el estudiante comience a descubrir su propia herencia, la de la cultura dominante y la del sistema de salud.

2. Concienciarse de la existencia de innumerables significados de los términos salud y enfermedad.

3. Analizar las creencias y prácticas de salud desde sus propias prácticas de autocuidado y su herencia familiar.

4. Desarrollar una profunda comprensión sobre el contexto y los aspectos sociológicos de los receptores de cuidados de salud.

5. Analizar en profundidad la cultura, filosofia e historia de los sistemas de salud alopáticos (modernos).

6. Analizar con detalle la cultura, filosofia e historia de los sistemas de salud homeopáticos (tradicionales).

7. Analizar con detenimiento determinados sistemas de salud etnoculturales (tradicionales).

Los conceptos clave proceden de diversas disciplinas, incluyendo entre otras:

- Antropología

- Educación médica básica

- Salud Ambiental

- Folklore

- Historia

- Inmigración

- Farmacologia

- Filosofía

- Salud Pública

- Política Social

- Sociología

- Teología

Las habilidades a desarrollar incluyen, entre otras:

- Valoración de la herencia

- Comunicación y uso de intérpretes

- Diagnóstico de salud de la comunidad y su empleo o Escucha

Las estrategias pedagógicas incluyen:

- Reforzar el valor de estos contenidos en los planes de estudios

\footnotetext{
2 (N. Del T.) Anne Fadiman, narra en su libro The Spirit catches you and you Fall Down, (1977) la historia de una familia y su confrontación con el sistema de salud americano cuando llevaron a su hija Lia a urgencias con convulsiones epilépticas. Para los padres "el alma de Lia había escapado de su cuerpo y se hallaba perdida". Conocían los síntomas como qaug dab peg, "el espíritu te atrapa y te caes". La familia no hablaba inglés, y en el hospital no había intérpretes. Se produjo una larga retahíla de malentendendidos y malinterpretaciones. El trágico conflicto cultural duró años y causó considerable dolor a ambas partes.

La autora cita este hecho en su libro Cultural Diversity in Health and Illness como ejemplo de lo que puede suceder cuando dos sistemas culturales de creencias chocan entre sí en el contexto de los sistemas de salud. Cada uno percibe y aborda el hecho con una idea preconcebida y surgen las dificultades, a menos que las partes sean capaces de comprender el punto de vista ajeno.
} 


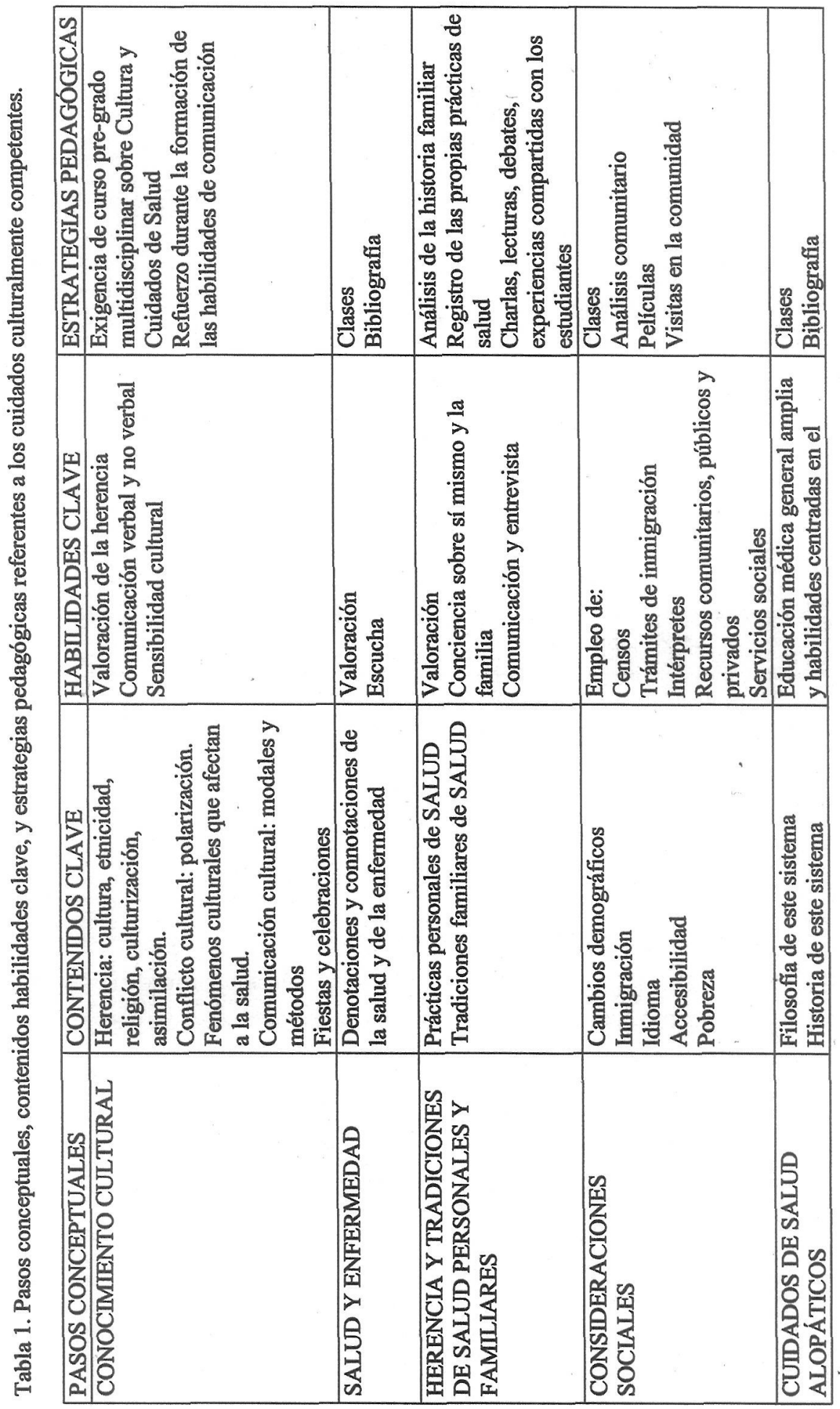


Cultura de los Cuidados • 9

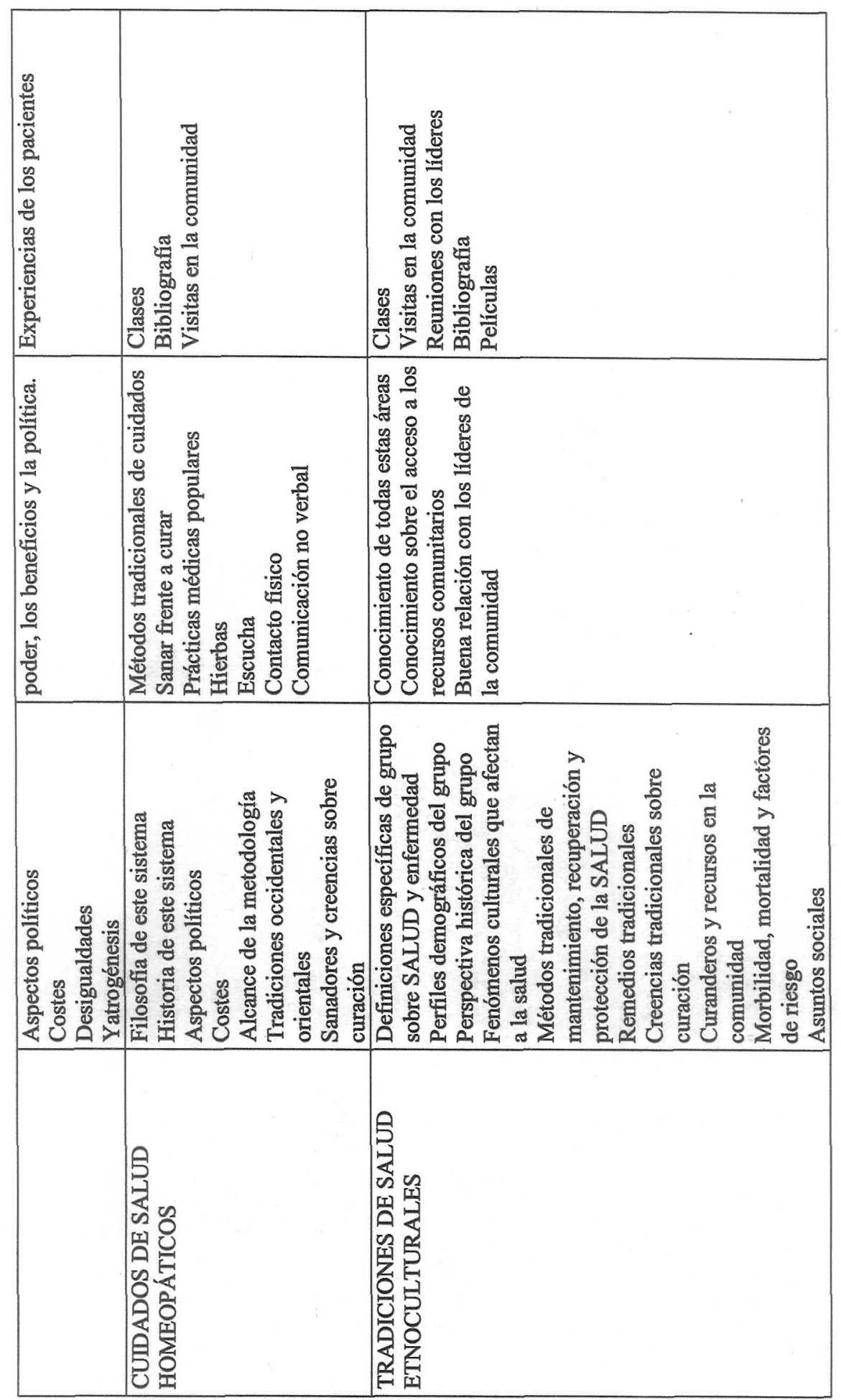


- Realizar análisis conjuntos sobre la comunidad

- Clases

- Bibliografía

- Charlas de representantes de algunas comunidades tradicionales

Se debe animar a los estudiantes a que, mientras estudian o trabajan, traten con personas de diversas comunidades. Una facultad de medicina que conozco estaba ubicada en la misma avenida que una botánica ${ }^{3}$. En ningún momento se instó a los estudiantes a que visitaran el establecimiento. No se tuvo en cuenta que en muchas comunidades hispanas las botánicas son a menudo el primer recurso de salud al que se acude. Los estudiantes deberian haber visitado este lugar y aprender sobre los cuidados de salud proporcionados alli.

Un plan de estudios tal que incluyera los Cuidados Culturales, podría revolucionar la educación y la práctica médicas puesto que se valorarian otras formas de cuidados de salud, y se desarrollaría el arte de la medicina con un toque humano. Desde el informe Flexner en 1910, el sistema alopático ha seguido en la línea de hacer primar la ciencia y la investigación empirica.

No quisiera concluir sin expresar mi más profundo desprecio, frustración y tristeza por los acontecimientos del pasado 11 de septiembre.

Tras dedicar mi vida profesional al desarrollo de los conocimientos basados en la cultura, esta tragedia ha hecho añicos la creencia de que la educación conduce a la comprensión, que a su vez lleva aparejados la armonía y los cuidados culturalmente competentes.

La polarización cultural que ha sido identificada a raiz de estos eventos incluye:

Fundamentalismo frente a pluralismo, herencia frente a secularismo, tradicionalismo frente a modernismo, minimalismo frente a exceso, SALUD 4 frente a salud, sanar frente a curar, homeopatia frente a alopatía, Etc.
De hecho, la investigación referente a estos conceptos y su repercusión inspirará numerosos esfuerzos educativos e investigadores. La idea es no abandonar, sino continuar avanzando y construyendo.

\section{BIBLIOGRAFÍA}

Estes, G. Y Zitzow, D. (1980) "Heritage Consistency as a Consideration in Counselling Native Americans". Ponencia presentada en la National Indian Association Convention, Dallas, Texas, noviembre.

Fadiman, A. (1977) The spirit catches you and you fall down. New York: Farrar, Straus, Giroux.

Giger, J.N. y Davidhizar, R.E. (1995) Transcultural Nursing Assessment and Intervention, $2^{\circ}$ edición. St.Louis: Mosby Yearbook.

Spector, R.E. (2000) Cultural Diversity in Health and Illness, 5 ' edición. Upper Saddle River, NJ: Prentice Hall.

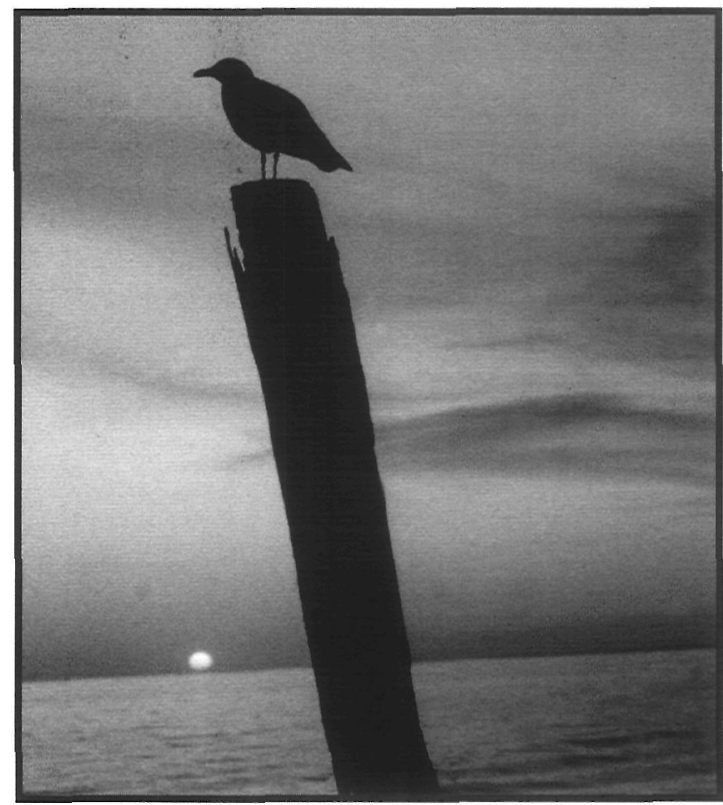

3 Botánica (N.del T.): Pequeño establecimiento tradicional en el que se adquieren todo tipo de remedios, principalmente hierbas, ungüentos e incienso (Spector 2000, Cultural Diversity in Health and Illness).

${ }^{4}$ (N.del T.) Tal y como explica en la introducción, la autora escribe SALUD con mayúscula cuando quiere decir salud holística entendida como equilibrio entre cuerpo, mente y espíritu (Spector 2000, Cultural Diversity in Health and IIlness) 